\title{
Dorsal Hand Vein Pattern Analysis and Neural Networks for Biometric Authentication
}

\author{
Bogdan BELEAN ${ }^{1}$, Mihaela STREZA ${ }^{1}$, Septimiu CRISAN ${ }^{2}$, Simina EMERICH ${ }^{3}$ \\ ${ }^{1}$ National Institute for Research and Development of Isotopic and Molecular Technologies, CETATEA \\ Research Centre, 67-103 Str. Donat, Cluj-Napoca, 400293, Romania \\ bogdan.belean@itim-cj.ro \\ ${ }^{2}$ Technical University of Cluj-Napoca, Department of Electrical Engineering and Measurements, 28 \\ Memorandumului, Cluj-Napoca, 400114, Romania \\ Septimiu.Crisan@ethm.utcluj.ro \\ ${ }^{3}$ Technical University of Cluj-Napoca, Department of Communications, 28 Memorandumului, Cluj-Napoca, \\ 400114, Romania \\ Simina.Emerich@com.utcluj.ro (*Corresponding author)
}

\begin{abstract}
Over the past several years, the subcutaneous blood vessels have emerged as a new solution for identity management. Biometric systems based on hand veins are considered to be very promising for high security environments. In this paper, we propose a novel user authentication approach based on dorsal hand vein pattern analysis and multi-layer perceptron neural network classification. For image processing two different techniques are employed: rotation invariant Hough transform and clustering based segmentation and mathematic morphology. Both approaches lead to binary images containing the vein patterns. The vessel structure corresponding to hand image samples of the same user are used to extract the final features, independent of hand rotation and distance to the camera lens during acquisition. These characteristics are used to train the neural network, whereas are computed for the new input images to be classified as corresponding to one of the legitimate subjects or not. The experimental analysis shows that user classification with an equal error rate of $0.83 \%$ can be attained, bringing the advantages of proposed image processing techniques for vein detection and neural network classification into complete synergy.
\end{abstract}

Keywords: Dorsal hand vein, Pattern recognition, Artificial neural network, and Authentication.

\section{Introduction}

In our contemporary information society, people expectations are to access private data anytime and anywhere. Passwords were generally used to secure information. Nevertheless, passwords can be guessed or forgotten, so biometric authentication is increasingly employed for security purposes. The main idea is to identify individuals through their unique biological information. For example, each subject has a unique pattern of its blood veins. If we take our hands into account, considering the broad and complex vascular pattern, the dorsal hand blood veins can be used as a promising feature for human authentication. The most important characteristics of the dorsal hand veins which make them suitable for biometric identification are: the vascular patterns do not vary during lifetime, the impossibility of being forgotten and the uniqueness of the veins shape among individuals.

Before proceeding to biometric identification based on vein recognition, a common procedure is to capture the infrared images (IR) of the hand which contains the patterns of blood vessels [17]. One can assume that a superficial vein acts as a microscopic thermal source located inside the dermis whose temperature depends mainly on the blood flow. A basic thermal model of an arm taking into account the contribution of a vein is described in [2]. It was shown that a modulated stimulation associated to a lock-in detection improves the imaging of the vessels, allowing the collection of veins radius and depth.

However, this scenario involves an IR camera for acquisition of thermal images, which increases the cost of the identification systems.

A low-cost solution would be the use of near infrared lighting to illuminate the dorsal area of the hand. Hemoglobin, in both arteries and veins strongly absorbs infrared radiation while water and the surrounding tissue do not. Veins are closer to the surface of the skin and due to the shallow penetration of infrared light (2-4mm), an infrared sensitive camera is able to capture the vein pattern while the arteries remain invisible. Biometric technology based on vascular recognition has been first developed for commercial purposes in 1997. Since then, many identification systems that utilize vascular patterns from different parts of the hand (finger, palm, dorsal side and wrist) have been introduced to the market, especially by Hitachi and Fujitsu [3].

Several methods proposed in literature for palm and dorsal hand vein pattern recognition and their 
use for biometric authentication are summarized next. For example, texture analysis is combined with a new shape representation method for dorsal hand veins pattern retrieval in [5] and [18]. Novel texture feature measurements, namely Centroid-based Circular Key-point Grid (CCKG), have recently been proposed in [6] for personal authentication through dorsal hand vein model. Local descriptors based on 2-D Gabor filters are employed in [9], for palm veins recognition. A similarity measure based on normalized Hamming distance is used for user authentication. Yan et al proposed in [19] an algorithm for user recognition based on image fusion technique applied on multiple palm image samples. They observed that it is difficult to obtain sufficient features for effective recognition using a SIFT algorithm on a single image. The image fusion technique brings up significant improvements in terms of user recognition performance. A supervised learning algorithm based on interactive artificial bee colony is used in [7] for collaborative representation of palm vein pattern using multiple images for the same user. Personal authentication is also successfully achieved using vein triangulation and knuckle shape in [8]. Matching scores are computed and authentication is performed with an equal error rate of $1.14 \%$. As suggested by previous studies [19], [7], image processing on single palm image often is not enough for user authentication. Fusion techniques and supervised learning are used on multiple sample images for the same user for efficient recognition. When multiple image samples are acquired and available for user authentication, feature extraction techniques combined with neural network (NN) based classification have been proved to be efficient for biometric identification [11].

Our work is focused on dorsal hand vein patterns examination and detection, particularly for the cases when it is difficult to achieve a satisfactory recognition performance with geometry and pixel intensity distribution - based methods. The major challenges are associated with nonuniform illumination, affine transformation and changes involved in hand positioning during acquisition process.

On one hand, for characterizing the vein pattern, an image processing pipeline is proposed, as a software tool and employed to identify the elongated dark patches, corresponding to the blood vessels area. Based on the detected dark patches and the linear Hough transform a set of parameters is generated, which uniquely characterize the vascular structure.

On the other hand, vein patterns are detected using clustering based segmentation and mathematic morphology. Based on the result delivered by the image processing procedures, a complex system is developed for authentication. Multiple image samples recorded from the same user together with results delivered by different image processing approaches are used to train the back propagation neural network, employed for user classification and, consequently, authentication.

The results provided by the considered classification approaches, Simple Pattern Matching Authentication (SPMA) and NN-based Authentication (NNA), are compared in terms of Equal Error Rate (EER).

The remainder of this paper is organized as follows. Section II introduces the proposed methods for vein pattern detection using linear Hough transform (VP-HT) respectively clustering based segmentation and mathematic morphology (VP-CSM). The techniques employed for user classification are described in Section III. Experimental results are presented and discussed in Section IV, and finally, conclusions are drawn in Section V.

\section{Methods}

The proposed methods are tested and evaluated using a public dataset, namely Bosphorus hand vein database [20]. The images were collected at Bogazici University, Turkey, by using a monochrome Near Infrared camera. Every image is stored as a bi-dimensional, 300×240 pixels array of intensities $I=\left(p_{x, y}\right)$. The intensities $p_{x, y}$ are 8 bits integers with a dynamic range of 0 $<p_{x, y}<2^{8}-1$, representing the pixels of the 256 gray levels digital image. Figure 1.a shows an example of a dorsal hand venous network. For each individual, 12 representations of the left hand have been captured, during four different session: under normal condition (Normal); after carrying a $3 \mathrm{~kg}$ bag, for one minute (Bag); after squeezing an elastic ball for one minute (Activity) and after cooling the hand in ice (Ice). For each session three images have been taken, with the intension of simulating the physical stress conditions that could appear in real-life situations [21]. 
Two approaches are further proposed for vein pattern recognition. In the first one, the rotation invariant veins detection based on the linear Hough transform (VP-HT) is performed and the determined line segments are used to construct the vascular pattern. The second approach makes use of the clustering based segmentation and mathematic morphology to detect the vein pattern (VP-CSM). Both methods lead to a binary image containing the dorsal hand vascular structure. The resulted image is stored as a bi-dimensional array of binary values, $V=\left(b_{i, j}\right)$ for both approaches. Nevertheless, the two proposed image processing methods do not lead to exactly same results. Since the image acquisition was performed using a digital camera in various environments, slight variation considering luminosity exists between different image captures. Moreover, veins position and their corresponding intensity in the image plane may also vary due to tense or relaxed finger muscles and blood pressure. Thus, subsequent palm image acquisition for the same user leads to slightly different results in terms of vein pattern, considering a given technique. Furthermore, the two proposed image processing approaches, also lead to slightly different vein pattern for the same user. In order to exploit the variations introduced by image acquisition process and by different image processing approaches when detecting the vascular pattern in dorsal hand, Section 3 proposes an artificial neural network model to perform user classification. The following sections present the proposed VP-HT and VP-CSM vein pattern recognition approaches.

\subsection{Vein pattern detection using linear Hough transform (VP-HT)}

The first proposed algorithm identifies veins in the images and extracts their characteristics, such as size, width and angle. Our workflow includes the following steps: (1) detection of palm edges, (2) pre-processing for enhancement of the veins within their corresponding area, (3) determination of the veins segments and their size, (4) rotation invariant determination of veins positions and (5) extraction of veins pattern biometric parameters.

\section{Palm edge detection}

The Canny edge detector is employed to extract the palm contour. This step includes a convolution of the input image with a Gaussian kernel of size 5 pixels and a standard deviation of $\sigma=4$. The selection of image pixels as part of the palm edges is based on a non-maximal suppression threshold equal to $70 \%$ of the maximum local gradient value.

The result can be seen in Figure 1.b. The purpose of the edge detection is to determine the reference line segment $[A B]$ used to compute the veins pattern biometric parameters. Let $c(x, y)$ be the curved line which determines the edge contour. The $[A B]$ segment is defined as the line segment given by the two points corresponding to the 2 nd and the 4 th valleys locations (i.e. concave points) of the determined edge profile. Note that veins positions, as referred to the previously defined line segment, are invariant to the palm rotation.

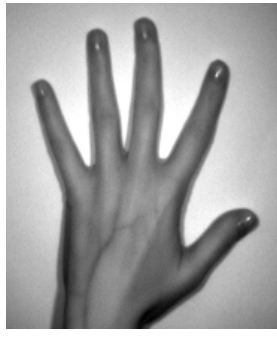

a)

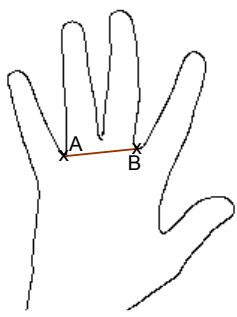

b)
Figure 1. a) Palm image, b) Detected palm edge, used for the $[A B]$ line segment identification

\section{Preprocessing for veins enhancement}

The current step includes both a point-wise transform and anisotropic diffusion for veins enhancement. Firstly, to the image $I$ stored as a bidimensional array $p_{x, y}$ of 8 bits integer intensities, a hyperbolic tangent transformation (tanh) maps the $p_{x, y}$ values to the real-valued interval $-1<$ $p_{x, y}<1$. Further on, a uniform scaling procedure yielded to a $\tan h$ transformed image $I^{\prime}=\left(p_{x, y}{ }^{\prime}\right)$ with enhanced veins pattern.

As seen in Figure 2.a, the hyperbolic tangent transform enhances or decreases the pixel intensities values relative to a threshold $k$. The $k$ threshold is adaptively chosen according to the background values. The background values (Figure 2.b) are locally estimated, based on the mathematic morphology (i.e. closing procedure followed by an opening procedure).

The anisotropic nonlinear diffusion model was proposed by Perona and Malik [10] for avoiding the blurring and localization problems, by applying an inhomogeneous process that reduces the diffusivity at those locations which have a larger likelihood to be edges. The diffusion is known as a physical process that equilibrates 
concentration differences without creating or destroying mass. The above equilibrium property, is given by the mathematical formulation:

$j=-D \cdot \nabla I^{\prime}$,

where $D$ is a diffusion tensor represented by a positive symmetric matrix, which establishes the relation between the concentration gradient $\nabla I^{\prime}$ and a flux $j$ which aims to compensate for this gradient. In case $j$ and $\nabla I^{\prime}$ are parallel, the diffusion is called isotropic. The property of diffusion of not destroying mass/information is expressed by the continuity equation (2).

$\partial_{t} I^{\prime}=-\operatorname{div} j$

Considering the above, the diffusion expression is given by equation (3), which appears in case of different transport processes.

$\partial_{t} I^{\prime}=\operatorname{div}\left(D \cdot \nabla I^{\prime}\right)$

Regarding the diffusion tensor, if it depends on the evolving image in time domain, the diffusion is called non-linear or anisotropic. Considering $I$ ' the input image, the probability for a specific area to be an edge is given by $s=\left|\nabla I^{\prime}\right|^{2}$. In order to reduce the diffusivity at those locations which have a larger likelihood to be an edge (i.e. in case of the veins patterns), the diffusion equation is written as:

$$
\partial_{t} I^{\prime}=\operatorname{div}\left(g\left(|\nabla \mathrm{I}|^{2}\right) \cdot \nabla I^{\prime}\right)
$$

The diffusivity $g$ is adapted to the image structure, being a function of the norm of the local gray level distribution gradient denoted by $s=\left|\nabla I^{\prime}\right|^{2}$. The diffusivity should be infinitely continuous differentiable and should decrease monotonically from 1 to 0 while $s^{2}$ varies from 0 to $+\infty$. In our case, when the purpose is to underline the veins patterns, the diffusivity is chosen as:

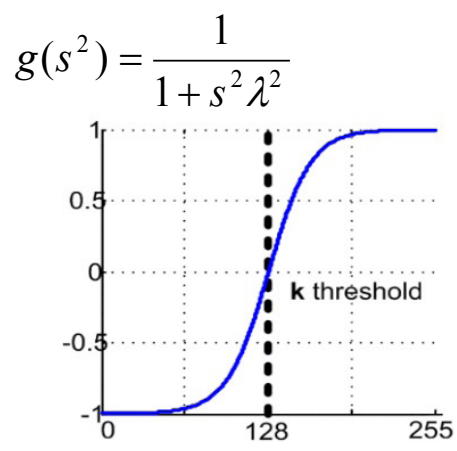

a)

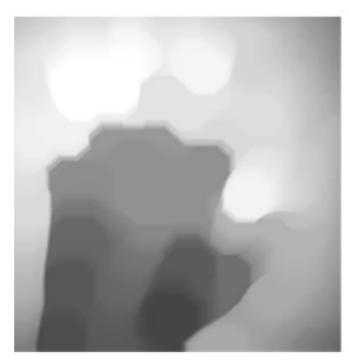

b)

Finite differences are used to approximate the equation (4), which lead to the discrete form of the anisotropic diffusion, which is characterized by an iterative procedure applied on the input palm image. The number of iterations corresponds to the $t$ parameter from equation (4).

The palm image from Figure 1.a is enhanced and filtered using anisotropic diffusion and the results are presented in Figure 2.c. The number of iterations for the anisotropic diffusion filter is 25. In order to privilege high-contrast edges over low-contrast ones, the parameter $\lambda$ is empirically selected to be 10 . For larger values of $\lambda$ details are lost. Comparing the Figure 1.a and the Figure 2.c, the effects of such transform after image enhancement are visually impressive; edges remained stable over an increased number of iterations used for the diffusion filter.

\section{Determination of the veins segments}

The principle of the Hough technique is based on a global description of image features, namely the number of pixels included in the feature solution classes are tagged using a voting mechanism within an accumulator.

In our case, aiming to detect image line segments, we describe a line segment by the following parametric equation:

$x \cos \theta+y \sin \theta=r$

For any $\left(x_{i}, y_{i}\right)$ point on this line, $r$ (the length of the normal from the image origin) and $\theta$ (the orientation of $r$ ) are constant. Consequently, the Hough transform for line segments is performed through the following steps: the gray-level image is processed by an edge detection filter, and an edge mask is produced. The image pixels included in the determined mask are denoted by $\left(x_{i}{ }^{\prime}, y_{i}{ }^{\prime}\right)$. Based on the determined edge mask, we verify for each pixel to which line it belongs and

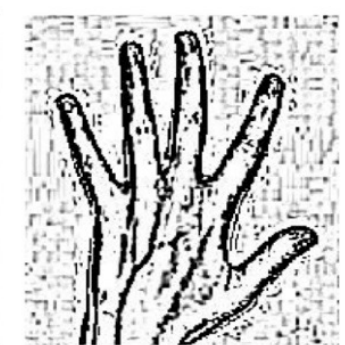

c)

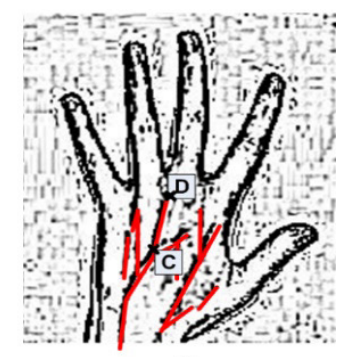

d)

Figure 2. Vein pattern detection using the adaptive Hough Transform: a) hyperbolic tangent function profile b) background estimation using mathematic morphology, c) enhanced vein pattern, d) detected vein segments 
we vote in the accumulator array to the position corresponding to the established line. After the end of the voting step, by a thresholding procedure we determined the lines within the image. Still, in case of veins parameter determination, this information is not sufficient. Thus, as referred to the original image, we are interested into the beginning and end of each line, which lead to the next processing step, line segment determination. This is performed through the determination of beginning and end points denoted by $B(x, y)$ and $E(x, y)$ respectively, for each line segment. $B(x, y)$ and $E(x, y)$ represent the points of intersection between the edge mask and the line specified by a $(r, \theta)$ pair. An example of a determined line segment $[C D]$ is presented in the Figure 2.d.

\section{Extraction of biometric vein features}

A simple rotation invariant vein pattern characterization is proposed next, based on the Hough-transform approach for veins segments determination. The parameters considered for vein pattern characterization are discussed further on. After applying the Hough transform, the dorsal hand vein pattern is characterized by a set of lines $v$, with $i$ from 1 to $n$, where $n$ represents the number of detected lines. Let us consider the line segment $[C D]$, the correspondent of one hand vein. It is characterized by the end points $C$ and $D$ and by the $(r, \theta)$ pair determined by the Hough transform. The position of the line segment is given by $r$ (i.e. the length from the image origin), while the line segment orientation is given by $\theta$. Note that, the $(r, \theta)$ pair depends on the palm rotation and also, on the distance between hand and camera lens. Thus, regarding the orientation of each line segment $v_{i}$ associated with hand veins, the angle $\theta$ ' between $[A B]$ and $[C D]$ line segments is used, in order to have a rotation invariant parameter. If we take into account the $v_{i}$ line segment position given by $r$, this is computed relative to the distance from the image origin to the $[A B]$ line segment. The previous computation leads to another value (i.e. $r$ ') which is invariant to the hand image origin. Note that the origin may differ from one hand image to another.

The third parameter used for biometric identification is the length $l$ of the veins, computed relative to the $d$ size of the [AB] segment. This also eliminates the erroneous estimation of veins size due to the distance between camera lens and hand, during image acquisition.

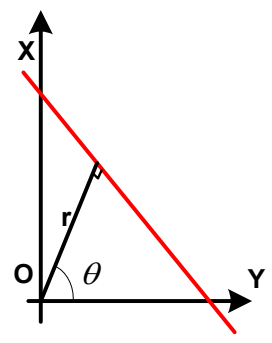

a)

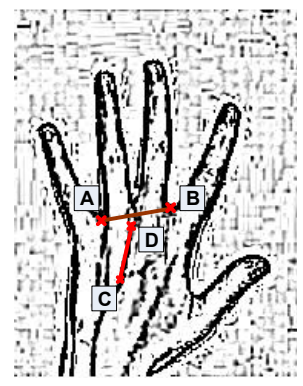

b)
Figure 3. Determination of the rotation invariant $(r, \theta)$ parameters for a given $[C D]$ line segment

The proposed approach leads to the $\left(r^{\prime}, \theta, l\right)$ triplet for each $v_{i}$ line segment. The whole set of parameters determined for the entire set of $n$ line segments characterize the hand vein pattern for each individual.

\subsection{Vein pattern detection using clustering based segmentation and mathematic morphology (VP-CSM)}

Up to this point, line segments corresponding to vessels are detected using the Hough transform. The complete set of segments form together the dorsal hand vein pattern for each individual user. Starting from the point wise hyperbolic tangent enhancement procedure, another approach is presented next for the vein pattern detection.

Let $I^{\prime}=\left(p_{x, y}\right)$ represents the input image with adaptively enhanced veins pattern. The VPCSM procedure for the vein pattern extraction is composed of three steps: (1) cutting the image section containing only the dorsal hand area, (2) initial classification of pixels by k-means clustering into background, veins, and outliers such as shadows, (3) refinement of the classification by morphological thinning. This procedure is iterative, thus, several successive steps (i.e. 3 to 5) representing morphological erosion are applied to the analyzed image. Thus, the result of the proposed procedure is stored as a bi-dimensional array of binary values, $V=\left(b_{i, j}\right)$, representing the veins pattern. The aforementioned approach is exemplified on the image presented in Figure 1.a. The resulted binary image $V$, containing the vein pattern is 
illustrated in Figure 4.a-c, where each of the 3 figures represents the binary image resulted at each iteration steps from 1 to 3.

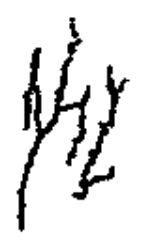

a)

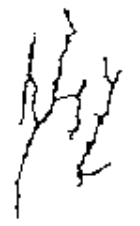

b)

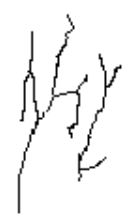

c)
Figure 4. Vein pattern detection using clustering based segmentation and mathematic morphology

\section{Data set and neural network approach for user classification}

For the same user, different vein patterns are acquired in cases of sequential acquisitions due to issues such as non-uniform illumination, affine transformation and changes involved in hand positioning between one acquired image and the next. Moreover, tense or relaxed finger muscles or blood pressure may introduce slight variations in the hand vein pattern. Taking into account all of the above, we design the experimental setup by using 60 images, from 15 users, as gallery set.. The question is how a biometric system can benefit from the results obtained by investigating the considered images with different processing techniques (i.e. VP-HT and VP-CSM. A neural network based classification represents a promising solution if user authentication is intended to be performed, using several images for each subject and different image analysing methods.

\subsection{Neural network based vein pattern classification}

We define a set $U=\left(u_{i}\right)$, with $i=1 \ldots N$, where $N$ represents the number of users to be further on authenticated by our neural network based biometric system (e.g. our experimental setup includes 15 users). The main principle of our approach is to train a multilayer neural network for the given set of dorsal hand images corresponding to the users included in $U$, which are part of our initial dataset. The neural network will further on classify any input image data as a genuine match with one of the users in $U$, or an impostor.

To describe the neural network based approach designed for user authentication, we define the input parameters and also the expected outputs. We consider 4 hand images stored for the user $u_{i}$. The two proposed methods, VP-HT and VP-
CSM, deliver one binary image $V=\left(b_{i, j}\right)$ each, representing the vein pattern for every input image. This leads to a number of 8 vein patterns $V$ for each $u_{i}$ user, which are further utilized for the neural network training. After the network training procedure, another $V_{c}$ vein pattern is delivered as input, and the networks will classify the input as corresponding to one of the 15 users or as an outlier (i.e. impostor). Consequently, for user recognition through vein patterns, a neural feed forward multi-layer perceptron (MLP) network with one hidden layer has been used. The network topology is given by each application specifications. In our case, the binary image $V$ of the vein pattern represents the input. Note that each image is partitioned into $p=4 \times 5$ sub-images (Figure 6) denoted by $V_{p}$ and the corresponding features are delivered as inputs to the MLP neural network. Consequently, 20 neurons belong to the input layer. The output layer is composed of $N$ $=15$ units (i.e. neurons), one for each user for which the authentication is performed. The output layer is used to represent the class scores (e.g. classification scores), which represent real numbers each giving the probability of the input image to be associated with one of the $u_{i}$ users. One hidden layer is considered for the neural network system. Ideally the number of hidden layer units is between $N$ and $2 N$, where $N$ is number of units in the output layer. Our choice is 30 units for the hidden layer. The node connections within network layers are unidirectional, leading to a feed forward topology. As for the activation function used by network elements, the sigmoid one is applied:

$$
\sigma(\mathrm{x})=1 /\left(1+\mathrm{e}^{-\mathrm{x}}\right)
$$

Considering the sigmoid function, the output of each neural network element (i.e. neuron) is given by equation (8). The aforementioned output can be interpreted as the probability for the input hand vein image to be associated with one of the $u_{i}$ users.

$$
P\left(u_{i}=1 \mid x_{i}, w\right)=\underset{i}{\sigma}\left(\sum_{i} w_{i} x_{i}+b\right)
$$

Note that, during image acquisition, the distance from the hand to the camera may vary from one session to another. Thus, for the same dorsal hand, the lengths of the same veins on two acquired images may be different, depending on the scaling factor introduced by the distance from the hand to 
the camera lenses. Consequently, a scale-invariant procedure is proposed for the features extraction. Other approaches consider image registration methods to find the best match between more images of the same scene captured in different conditions or by different sensors. For example, in [14] a bi-resolution algorithm is proposed for retinal image registration.

\subsection{Scale-invariant features computation}

We've seen so far that $V_{p}$ images are delivered as features to the neural network inputs. Note that the $V_{p}$ image, with $p=1$ to 20 , corresponds to the $p$ partition of the $V$ input image as shown in Figure 6. Further on the computation-procedure for each $x_{p}$ feature associated with the $V_{p}$ image is detailed. The distance between the capture device (digital camera) and the hand is considered for feature estimation. Thus, for each $V_{p}$ image, the total number of pixels $n$ which are part of the hand veins are computed and referred to the half perimeter size of the sub image $\mathrm{V}_{\mathrm{p}}$ according to $n_{r}=2 n / P$, where $n_{r}$ denotes the relative number of pixels from the sub-image $V_{p}$ and $P$ denotes the size in pixel of the $V_{p}$ image perimeter. Further, it comes natural to encode within the $x_{p}$ feature the position of the veins within the $V_{p}$ sub-image. Thus the following approach is used: firstly, the intersection points within the vein pattern and subimage perimeter are detected; for each intersection point $I$, the abscissa value and the ordinate value for points on vertical and horizontal perimeter lines respectively, are used as weights (i.e. $d_{i}$ distanclinees, where $I$ represents the number of intersection points of the vein patterns with sub-image perimeter) in $x_{p}$ feature computation. An example is given in Figure 6.b, where the $x_{p}$ feature is given by:

$x_{p}=(1+d 1+d 2+d 3) n_{r}$

and each $d_{i}$ takes values between 0 and 1 . An intersection point close to the sub-image origin $O$ leads to a low weighted distance $d_{i}$. The weight distance increases as the intersection point gets further from the sub-image origin. Hand images from 15 users are involved to validate the proposed neural network. A number of 4 images per user, acquired under different conditions, together with the two different techniques proposed for vein pattern detection are used to train the network.

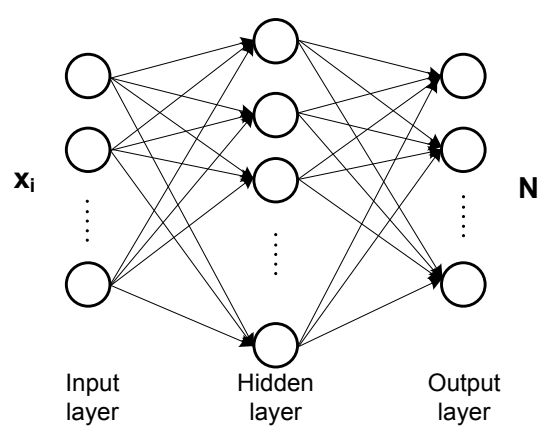

Figure 5. Neural network topology

Considering that for the mentioned set of input images, the output results are known (to which user $u_{i}$ corresponds the input image $V$ ), the gradient descent rule is used as an iterative error minimization method to adjust the neural networks weights.

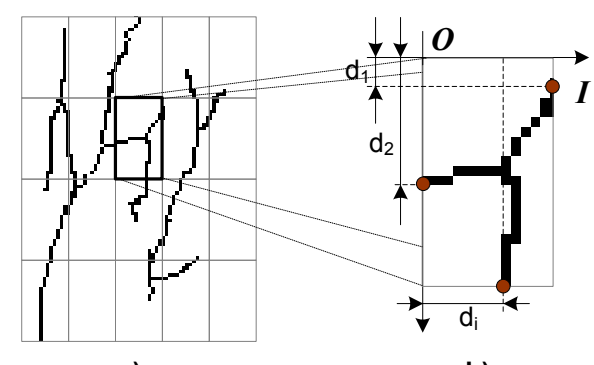

a)

b)

Figure 6. Input palm image partitioning and $x_{p}$ feature computation

Once the neural network is trained, the classification can be performed for any new palm image $V_{N}$ applied as input.

\section{Results and discussion}

As pointed out in [12], [13] and [16], classification tasks can be achieved by building the appropriate neural network model. In the proposed neural network based system, 60 images, corresponding to 15 genuine users (4 left hand images/user) are considered to be the gallery set. For each individual, one image has been captured under normal conditions (Normal) and three under stress (Bag, Activity and Ice).

The extracted vein pattern features are computed using the two image processing approaches (i.e. VP-HT and VP-CSM) and feed into the neural network for training purposes. Once the training is finished, vein pattern features from different users are computed and delivered to the neural network for authentication. The performance of 
the proposed biometric system is evaluated by conducting the experiments on 360 test images acquired from 45 users ( 8 samples/subject, 2 from each subset Normal, Bag, Activity and Ice). Thus, from the total number of 45 users, assumed as probe set, 15 are genuine users whereas the rest of 30 are considered intruders or impostors. The workstation used for experimentation is equipped with an Intel i5 processor, $2.5 \mathrm{GHz}, 4 \mathrm{~GB}$ of RAM memory, and Matlab 7.1 for algorithms implementation.

Further on, two authentication approaches are considered for evaluation, namely Simple Pattern Matching Authentication (SPMA) and NN-based Authentication (NNA).

The first authentication approach, SPMA, is based on the $\left(r^{\prime}, \theta, l\right)$ triplets detected by the VP-HT technique for each line segment and is evaluated based on a minimum distance. The normalized differences of the triplets' values, between the user to be authenticated and all the legitimate users are computed. The identity of the tested user is assigned to the closest database template if the resulted distance is lower than preset threshold $\left(k_{1}\right)$; otherwise it is considered to be an imposter.

In the case of the second approach, NNA, a decision of a matched or unmatched for a given user is taken based on a threshold, $k_{2}$ and a score given by the probability $P(u)$ for the input dorsal hand vein image to be associated with one of the $\underline{u}_{i}$ legitimate users. If the probability score $P$ is greater than $k_{2}$, then it indicates a match, while an unmatched is indicated by a $P$ score lower than $k_{2}$.

The False Acceptance Rate (FAR) that indicates the percentage of invalid inputs incorrectly accepted and the False Rejection Rate (FRR) expressing the percentage of valid inputs incorrectly rejected are defined in terms of $k_{1}$ and $k_{2}$ [1]. In this manner, the threshold $k_{i}$ defines the security level. A Receiver Operating Characteristic (ROC) curve can be used to see the behaviour of FAR and FRR at different threshold values. In Figure 7, the green line illustrates the $R O C$ curve obtained for the biometric system based on NNA. In order to underline the benefits of using the proposed neural network approach for user authentication, a similar $R O C$ curve (blue line) is plotted for the SPMA approach, based on the $\left(r^{\prime}, \theta^{\prime}, l\right)$ triplets. Further on, Equal Error Rate (EER) is used as the evaluation metrics for both the NNA and SPMA biometric systems. EER is given by the False Acceptance Rate at a particular threshold $k_{i}$, such that $F A R\left(k_{i}\right)=F R R\left(k_{i}\right)$. The proposed NNA system has achieved an EER of $0.83 \%$, significantly lower than SPMA approach $(\mathrm{EER}=1.67 \%)$.

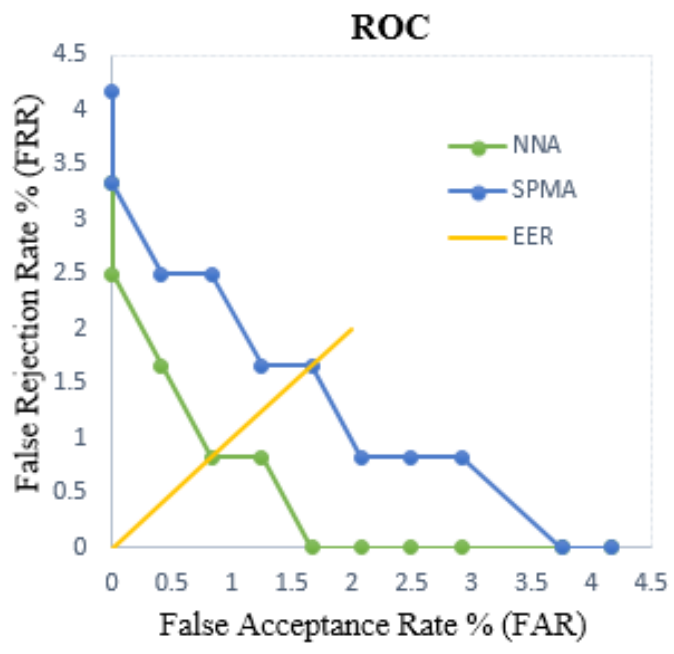

Figure 7. ROC curve of the proposed authentication systems based on NNA (green line), respectively SPMA (blue line)

The equal error rate of EER $=0.83 \%$ (NNA system) has been obtained for the security level $k_{2}$ equal with 0.81 . In this case, two test images belonging to impostors have been incorrectly accepted and one image corresponding to an authorized person has been rejected.

Table 1 presents comparative results of prior approaches, presented in literature for biometric recognition based on the veins from the back surface of the hand. An accurate comparison is quite difficult to achieve, because a biometric system performance is influenced by many factors: the number of enrolled users, the number of images considered as gallery respectively probe set, the quality of the images, etc.

Our proposed NN-based system proved to be suitable for a reduced number of genuine users. The question that arises is what happens with such system in case of hundred or even more subjects. Obviously, regarding the NN network topology, the number of nodes of the output layers should be increased. This leads to an increased number of features which need to be delivered to the input layer for efficient classification. Consequently, complementary solutions could be added to the proposed system in order to determine supplementary features for the $\mathrm{NN}$ input layer. 
Table 1. Comparative summary of related work on dorsal hand veins based biometric systems

\begin{tabular}{|c|c|c|c|c|}
\hline System & \multicolumn{2}{|c|}{ Methodology } & Database & EER \\
\hline $\begin{array}{l}\text { Kumar et al. } \\
(2009)[8]\end{array}$ & \multicolumn{2}{|c|}{ Matching vein triangulation and shape features } & Personal database & $1.14 \%$ \\
\hline $\begin{array}{l}\text { Uriarte-Antonio } \\
\text { et al. }(2011)[15]\end{array}$ & \multicolumn{2}{|c|}{ Minutiae extraction algorithm } & Singapore & $1.63 \%$ \\
\hline $\begin{array}{l}\text { Yuksel et al. } \\
(2011) \text { [21] }\end{array}$ & \multicolumn{2}{|c|}{$\begin{array}{l}\text { Fusion of geometric and appearance-based } \\
\text { techniques }\end{array}$} & Bosphorus & $1.98 \%$ \\
\hline \multirow{2}{*}{$\begin{array}{l}\text { Huang et al. } \\
(2015)[4]\end{array}$} & \multirow{2}{*}{\multicolumn{2}{|c|}{ Multi-source keypoint based SIFT matching }} & NCUT Part A & $0.81 \%$ \\
\hline & & & NCUT Part B & $1.12 \%$ \\
\hline \multirow[b]{2}{*}{ Ours } & \multirow{2}{*}{$\begin{array}{l}\text { Adaptive Hough } \\
\text { transform and } \\
\text { clustering } \\
\text { segmentation } \\
\text { and mathematic } \\
\text { morphology }\end{array}$} & SPMA k $_{1}=0.76$ & Bosphorus & $1.67 \%$ \\
\hline & & $\mathrm{NNA} \mathrm{k}_{2}=0.81$ & Bosphorus & $0.83 \%$ \\
\hline
\end{tabular}

\section{Conclusions}

In this paper we have proposed, discussed and tested a user authentication system based on neural network classification of dorsal hand vein pattern images. Two different image processing techniques, namely adaptive Hough transform (VP-HT) and clustering segmentation and mathematic morphology (VP-CSM), has been investigated for the same objective - vascular structure detection. Consequently, similar vein patterns are obtained for multiple hand image samples of the same subject. In this manner, for the same user multiple sets of hand features are achieved and used for the neural network training. After the network training, feature vectors corresponding to new palm images are sent to the matching module, aiming biometric authentication. Hand images captured under normal conditions as well as hands that underwent ice, bag and ball stresses have been included for both training and testing data sets. The Equal Error Rate has been reported as a performance indicator of the classification efficiency. According to the experimental results, the proposed system is comparable or even outperforms prior approaches for dorsal hand veins biometric recognition.

As future work, we intend to adapt the system so that to ensure the enrollment of a larger number of users.

In addition, ordinal measurement based techniques will be proposed for the image feature extraction module.

\section{Acknowledgements}

This work was supported by a grant of the Romanian National Authority for Scientific Research and Innovation, CNCS - UEFISCDI, project number PN-II-RU-TE-2014-4-2080.

\section{REFERENCES}

1. Bolle, R. M., Ratha, N. K., \& Pankanti, S. (2004). Error Analysis of Pattern Recognition Systems - The Subsets Bootstrap. Computer Vision and Image Understanding, 93(1), pp. 1-33.

2. Boué, C., Cassagne, F., Massoud, C., \& Fournier, D. (2007). Thermal Imaging of a Vein of the Forearm: Analysis and Thermal Modelling. Infrared Physics \& Technology, 51(1), pp. 13-20.

3. Choi, A. H., \& Tran, C. N. (2008). Hand Vascular Pattern Technology. In Handbook of Biometrics, Springer US, pp. 253-270.

4. Huang, D., Tang, Y., Wang, Y., Chen, L., \& Wang, Y. (2015). Hand-Dorsa Vein Recognition by Matching Local Features of Multisource Keypoints. IEEE Transactions on Cybernetics, 45(9), pp. 1823-1837.

5. Huang, D., Zhang, R., Yin, Y., Wang, Y., \& Wang, Y. (2017). Local Feature Approach to Dorsal Hand Vein Recognition by CentroidBased Circular Key-Point Grid and FineGrained Matching. Image and Vision Computing, 58, pp. 266-277. 
6. Huang, D., Zhu, X., Wang, Y., \& Zhang, D. (2016). Dorsal Hand Vein Recognition via Hierarchical Combination of Texture and Shape Clues. Neurocomputing, 214, pp. 815-828.

7. Joardar,S., Chatterjee, A., Bandyopadhyay, S., \& Maulik, U. (2017). Multi-Size Patch Based Collaborative Representation for Palm Dorsa Vein Pattern Recognition by Enhanced Ensemble Learning with Modified Interactive Artificial Bee Colony Algorithm. Engineering Applications of Artificial Intelligence, 60, pp. 151-163.

8. Kumar, A., \& Prathyusha, K. V. (2009). Personal Authentication Using Hand Vein Triangulation and Knuckle Shape. IEEE Transactions on Image processing, 18(9), pp. 2127-2136.

9. Lee, J. C. (2012). A Novel Biometric System Based on Palm Vein Image. Pattern Recognition Letters, 33(12), pp. 1520-1528.

10. Perona, P., \& Malik, J. (1990). Scale-Space and Edge Detection Using Anisotropic Diffusion. IEEE Transactions on pattern analysis and machine intelligence, 12(7), pp. 629-639.

11. Perwira, D. Y., Agung, B. T., \& Sulistiyo, M. D. (2014), Personal Palm Vein Identification using Principal Component Analysis and Probabilistic Neural Network. International Conference on Information Technology Systems and Innovation, pp. 99-104.

12. Salameh, W. A. (2004) Detection of Intrusion Using Neural Networks: A Customized Study. Studies in Informatics and Control, 13(2), 135-143.

13. Sifaoui, A., Abdelkrim, A., Alouane, S., \& Benrejeb, M. (2009). On New RBF Neural Network Construction Algorithm for Classification. Studies in Informatics and Control, 18(2), pp. 103-110.
14. Tuba, E., Tuba, M., \& Dolicanin, E. (2017). Adjusted Fireworks Algorithm Applied to Retinal Image Registration. Studies in Informatics and Control, 26(1), pp. 33-42.

15. Uriarte-Antonio, J., Hartung, D., Pascual, J. E. S., \& Sanchez-Reillo, R. (2011, October). Vascular Biometrics Based On A Minutiae Extraction Approach. In IEEE International Carnahan Conference on Security Technology (ICCST), pp. 1-7

16. Vrejoiu, M. H. (2017). A Pattern Matching Method and Algorithm for Face Detection. Studies in Informatics and Control, 26(1), pp. 75-86.

17. Wang, L., Wang, L., Leedham, G., \& Cho, D. S. Y. (2008). Minutiae Feature Analysis for Infrared Hand Vein Pattern Biometrics. Pattern recognition, 41(3), pp. 920-929.

18. Wu, K. S., Lee, J. C., Lo, T. M., Chang, K. C., \& Chang, C. P. (2013). A Secure Palm Vein Recognition System. Journal of Systems and Software, 86(11), pp. 2870-2876.

19. Yan, X., Kang, W., Deng, F., \& Wu, Q. (2015). Palm Vein Recognition Based on Multi-Sampling and Feature-Level Fusion. Neurocomputing, 151, pp. 798-807.

20. Yuksel, A., Akarun, L., \& Sankur, B. (2010). Biometric Identification Through Hand Vein Patterns. International Workshop on Emerging Techniques and Challenges for Hand-Based Biometrics, pp. 1-6.

21. Yuksel, A., Akarun, L., \& Sankur, B. (2011). Hand Vein Biometry Based on Geometry and Appearance Methods. IET computer vision, 5(6), 398-406. 